\title{
Quantitative genetic models of female choice based on "arbitrary" male characters
}

\section{Lorraine Heisler*}

Institute of Animal Behavior, Rutgers University, 101 Warren Street, Newark, New Jersey 07102 U.S.A.

Multivariate, quantitative genetic models are developed for the evolution of female mating preferences in situations where males contribute only their gametes to their progeny. Although female mating preferences may not be directly subject to selection, they can evolve via genetic correlations with other characters that are undergoing evolutionary change. The first set of models examines the evolutionary origin of mating preferences directed at one or more traits that may or may not be expressed only in males. When several selected characters possess additive genetic variance, an indirect selective force exists for the evolution of multivariate mating preferences. The magnitude of this force is proportional to the covariance between a female's relative preference for a given male's phenotype and the expected viability of his progeny. The contribution of any single character to this covariance determines its potential value as a mate choice criterion. The pattern of genetic and phenotypic covariation may cause selectively unimportant traits to be useful in mate choice. In the extrene, selectively neutral characters may become the objects of mating preferences, if they are relatively immune to random environmental variation and genetically correlated with selectively important characters. The second set of models examines the dynamic evolution of such a selectively neutral ("arbitrary") character that is both the object of a mating preference and genetically correlated with a third trait that affects viability. The outcome of evolution in this three character system is highly indeterminate. As in other sexual selection models, there exists a line of neutral equilibria wherein the mean of the criterion character matches the mean level of mating preference within the population, while the viability trait equilibrates at the phenotypic value conferring maximum viability. This line of equilibria, however, is not likely to be stable unless females choose mates according to absolute mating preferences. Thus, mating preferences that initially may arise as a mean of increasing offspring viability may nevertheless lead to indeterminate and potentially maladaptive evolutionary outcomes.

\section{INTRODUCTION}

Sexual selection by "female choice" is currently experiencing widespread popularity as a subject in evolutionary research. The existence of female mating preferences is now well-documented, and researchers are redirecting their attention to the troublesome problem of their explanation (e.g., Bateson, 1983). Most contentious is the evolution of female choice when males do not contribute directly to their mates or their progeny (via parental investment, territory defense, nuptial "gifts" and so forth). In such cases, the absence of direct selection for female mating preferences implies that selective processes can only lead to the evolution of mating preferences through linkage disequilibrium, specifically through nonrandom associations (genetic covariance) between alleles

* Present address: Department of Biology, Vassar College, Poughtkeepsie, New York 12601, U.S.A. promoting a mating preference and alleles conferring high relative fitness. In this way female mating preferences evolve as a correlated response to selection on other characters.

This explanation of female mating preferences implies that when direct effects of mate choice on female viability or fertility cannot be found we should nevertheless observe a correlation between the relative attractiveness of a male and his breeding value for one or more components of fitness. This empirical question has not yet been resolved (but see recent work by Partridge, 1980; Schaeffer, et al., 1984; and Boake, 1985). Meanwhile, a theoretical debate has arisen concerning which of the three major components of fitness-viability, fertility or mating success-are most likely to be the correlates of female choice. One side of this debate has come to be called the "good genes" 
hypothesis. Supporters of this view argue that female choice should largely be directed at characters that reflect a male's breeding value for viability or fertility (Trivers, 1976; Borgia, 1979; Wittenberger, 1983). This notion is rejected by supporters of the "runaway" process originally articulated by Fisher (1958). Proponents of this view argue that mating preferences can evolve solely through their association with characters that confer high mating success in males, which in turn results from the existence of the mating preference itself. In this view, male attractiveness may be unrelated or even negatively correlated with other components of fitness. At present, theoretical population genetic models largely support the runaway theory (O’Donald, 1967; 1980; Lande, 1981; Kirkpatrick, 1982).

The aim of this paper is to propose a theoretical argument for the relationship between the "good genes" and "runaway" theories. In particular, I develop two related models, the first showing how "good genes" can play an instrumental role in the evolutionary origin of mating preferences, the second showing how Fisher's "runaway" process may dominate the outcome of this evolution. The models are derived from earlier models of Lande (1981) and Heisler (1984). They differ from these, however, in that they consider the evolution of "arbitrary" mating preferences, that is, preferences directed at characters that may not themselves be the cause of variation in fitness, but merely reflect that variation owing to genetic correlations with other, directly selected, characters. Mating preferences of this sort occur frequently (though not always explicitly) in discussions of female choice, especially in discussions of the complex and idiosyncratic characters that comprise speciesspecific courtship (e.g., vocalisations, chemical signals and courtship "dances"). Many such behaviours must themselves have been selectively neutral at the time mating preferences for them arose, and must subsequently have experienced direct effects largely from sexual selection, having few or no consequences for relative survivorship. (For example, the courtship characters used in species-recognition are almost certainly not themselves the cause of reduced viability and fertility in hybrids).

The idea that mating preferences can arise for characters that have no direct effect on fitness, but rather serve as a "guide" to it, was lucidly outlined by Fisher (1915), and the present models attempt a mathematical representation of his theory. They will be developed in the following sequence. First, I review my assumptions of poly- genic inheritance and optimising natural selection used throughout. Second, I develop a general multivariate model for the relationship between variation in male "genetic quality" and a selective force promoting the origin of a novel mating preference. This mating preference may itself be multivariate, involving simultaneous assessment by females of multiple characters in potential mates. Third, I analyse in detail the special case in which a mating preference directed at a selectively neutral trait may originate within a population. Finally, I present a dynamic model for the joint evolution of a female mating preference with two correlated characters, one that is subject only to intersexual selection acting on males, the other subject only to viability selection which may or may not be sex-limited.

\section{ASSUMPTIONS}

The models make the conventional assumptions used in modelling the evolution of multiple, continuously-varying phenotypic characters (e.g., Lande, 1979; 1980). Female mating preferences may be directed toward one or more quantitative characters in males. Any individual is characterised by a column vector of phenotypic values, $z=\left(z_{1}, z_{2}, \ldots, z_{n}\right)^{T}$, where the superscript " $T$ " denotes transposition. Each entry of $z$ is assumed to be equal to the sum of additive effects from alleles at a number of autosomal, diploid loci, plus an uncorrelated random effect of the environment. I assume that on some scale of measurement the distributions of additive genotypic values and environmental deviations are each multivariate normal, with nonsingular covariance matrices $\boldsymbol{G}$ and $\boldsymbol{E}$, respectively. If the mean of environmental effects is zero for all characters and there are no genotype-environment interactions, the distribution of phenotypic values is also multivariate normal, with vector of mean phenotypic values, $\bar{z}$, equal to the vector of mean genotypic values, and phenotypic covariance matrix $\boldsymbol{P}=\boldsymbol{G}+\boldsymbol{E}$.

Viability selection on any given trait is assumed to be either absent or optimising, with $w(z)$, the relative viability of an individual having vector, $z$, of phenotypic values, given by,

$$
w(z)=\exp \left(-{ }_{2}^{1} z^{\top} \boldsymbol{W} z\right),
$$

where $\boldsymbol{W}$ is a positive semidefinite diagonal matrix. Selection on the phenotypic variances is assumed to be weak (eigenvalues of $\boldsymbol{W}$ much less than those of $\left.\boldsymbol{P}{ }^{1}\right)$. Note that in the formulation of equation (1), the optimum phenotype under viability 
selection is the zero vector; thus, phenotypic values are scaled to measure the magnitude and direction of individual deviations from the optimum phenotype.

Two patterns of selection will be considered. In the first, the characters are assumed to be identically distributed and subject to the same pattern of viability selection in both sexes. In the second, viability selection is assumed to act only on males. Following selection, the distribution of phenotypic values within the selected portion of the population is, in either instance,

$$
p^{*}(z)=\frac{p(z) w(z)}{\int p(z) w(z) d z},
$$

where $\int d z$ denotes $\int_{-\infty}^{\infty} \cdots \int_{-\infty}^{\infty} d z_{1}, \ldots, d z_{n}$, and $p(z)$ is the phenotypic distribution before selection. This distribution is multivariate normal, with covariance matrix,

$$
\boldsymbol{P}^{*}=\left(\boldsymbol{P}^{-1}+\boldsymbol{W}\right)^{-1},
$$

and vector of mean phenotypes,

$$
\overline{\boldsymbol{z}}^{*}=\boldsymbol{P}^{*} \boldsymbol{P}^{-1} \overline{\boldsymbol{z}}
$$

Evolutionary change in the mean phenotypes is given by,

$$
\Delta \bar{z}=k \boldsymbol{G} \boldsymbol{P}^{-1}\left(\bar{z}^{*}-\bar{z}\right),
$$

where $k=\frac{1}{2}$ if selection is sex-limited, and $k=1$ if it is not. In either case, evolutionary equilibrium under this type of selection occurs only when $\bar{z}_{i}=0$, for all characters. (A detailed analysis of this model is given in Lande (1979).)

I assume that female mating preferences are never subject to direct selection. Hence, mating preferences can only evolve as a correlated response to selection on other characters. Mating preferences are modelled using a multivariate generalisation of the mating preference functions introduced by Lande (1981). In general, mating preferences are treated as weighting systems that alter the probability distribution of a female's mates from that expected under random mating. If more than one character is assessed by females during mate choice, these characters must somehow be combined into a scalar measure that describes a female's net preference for a male having any particular set of character values. I refer to such weighting systems as "preference index functions" and denote them $\Psi(z \mid y)$. In this formulation, $\boldsymbol{y}$ is a vector of weighting parameters that may differ from female to female; it is an individual female's "preference phenotype". Thus, female preference depends both on the particular values, $y_{1}, \ldots, y_{n}$, that a female assigns to the different male characters, and on the way in which these combine via the preference index function to create a net preference for any particular male. Four different forms of the function, $\Psi(z \mid y)$, will be considered in the following models.

\section{MODELS FOR THE ORIGIN OF A MATING PREFERENCE}

\section{(i) General Multivariate Model}

Here I develop a model that describes how selection on multiple phenotypic characters creates a force promoting the evolutionary origin of mating preferences. My aim is to show how genetic correlations between characters can affect the "suitability" of different traits as mate choice criteria.

It is a truism to state that any female mating preference must, at some point, enter a population that previously lacked it. To consider the selective forces operating at such a time, I assume here that mating within the focal population is random with respect to the characters being considered, and that viability selection is the only significant force acting on them. I further assume that the genetic loci that determine criterion (preferred) traits are in linkage equilibrium (randomly associated) with the loci that determine female mating preferences. If this is so, then the only deterministic process that can lead to the increase in frequency of an initially rare mating preference is the evolution of genetic correlations (linkage disequilibrium) between the mating preference and characters that confer high viability. Whether or not such genetic correlations will evolve can be evaluated by considering the expected effect of a particular pattern of mate choice on the viability of a female's progeny. (For a detailed discussion of the rationale of this approach, see Heisler, 1984.)

Let $\bar{w}^{\prime}(z)$ denote the expected fitness of the progeny of a male hving vector, $z$, of phenotypic values. Consider a mutant female having preference phonotype, $\boldsymbol{y}$, entering a randomly mating population. The relative preference of this female for mating with a male of phenotype $z$ denoted $\Psi^{*}(z \mid y)$, is obtained by normalising her absolute preference given the distribution of available mates:

$$
\Psi^{*}(z \mid y)=\frac{\Psi(z \mid y)}{\int p^{*}(z) \Psi(z \mid y) d z} .
$$

The distribution of this female's mates is assumed to be determined jointly by her preference and the 
availability of different types of males as the product: $p^{*}(z) \Psi^{*}(z \mid y)$. By definition then, the expected viability of progeny of this female is,

$$
E\left\{\bar{w}^{\prime}(z) \mid y\right\}=\int p^{*}(z) \Psi^{*}(z \mid y) \bar{w}^{\prime}(z) d z .
$$

On the other hand, the expected viability of the progeny of the randomly mating females in the population is

$$
E\left\{\bar{w}^{\prime}(z)\right\}=\int p^{*}(z) \bar{w}^{\prime}(z) d z .
$$

Letting $F(y)$ denote the change in expected progeny fitness of the novel, "choosy" female relative to that of the remainder of the females in the population, then,

$$
\begin{aligned}
F(y) & =E\left\{\bar{w}^{\prime}(z) \mid y\right\}-E\left\{\bar{w}^{\prime}(z)\right\} \\
& =k \operatorname{cov}\left\{\bar{w}^{\prime}(z), \Psi^{*}(z \mid y)\right\} .
\end{aligned}
$$

I have shown elsewhere (Heisler, 1984) that $F(y)$ is equivalent to a fertility selection coefficient if the population is censused as breeding adults. Equation (8) thus states that so long as a female's phenotype, $\boldsymbol{y}$, induces a positive covariance between her relative preference for a male and the expected viability of this progeny, selection will lead to an increase in the frequency of alleles for a mating preference. The factor of $k$ again shows the effect of sex-limitation of viability selection, with $k=\frac{1}{2}$ if selection is sex-limited and $k=1$ if it is not. Naturally, sex-limitation of viability selection reduces the indirect advantage of mate choice, since in that case only a fraction of a female's progeny experience differential viability.

Equation (8) reveals a formal similarity, between the situation confronted by females in choosing mates and that confronted by human applied geneticists in constructing "selection indices" for multiple characters in artificially selected stocks. In both cases, a variety of traits are evaluated and converted into some net measure, such as $\Psi(z \mid y)$, which forms the basis for selection of individuals. The object is to construct an index that maximises, for a given level of culling (or "choosiness") the increase in expected "worth" of progeny. In both cases, the covariance between predicted worth, $\Psi^{*}(z \mid y)$, and realised worth, $\bar{w}^{\prime}(z)$, provides a measure of the efficacy of $y$, the weighting system used (Bulmer, 1980). Thus, in the case of female mating preferences, the optimum mate choice "index" is that which maximises the covariance in equation (8). This optimum index characterises the magnitude and direction of the indirect force promoting the evolutionary origin of a mating preference. It does not, however, imply that "optimal mate choice" will evolve. It will become evident upon further development of the model that while a positive covariance in equation (8) creates a "good genes" effect, it does not lead to the consequences frequently credited to this mechanism.

Examination of the simplest type of directional mating preference, a set of linear weights attached to the different male characters, reveals several basic features of the formulation in equation (8). Consider the linear preference index function:

$$
\Psi(z \mid y)=1+y^{T}\left(z-z^{*}\right)=\Psi^{*}(z \mid y) .
$$

In this model, preference is relative to the mean of available males, with a value of unity assigned to phenotypically average individuals. Substitution of (9) into (8) yields,

$$
\begin{aligned}
F(y) & =k y^{T} \operatorname{cov}\left\{z, \bar{w}^{\prime}(z)\right\} \\
& =k \sum_{i} y_{i} \operatorname{cov}_{i}\left\{z_{i}, \bar{w}^{\prime}(z)\right\} .
\end{aligned}
$$

Thus, the initial selective advantage of a linear mating preference is a weighted sum of the weights, $y_{i}$, that a female attaches to the separate male characters. For a fixed level of female discrimination (i.e., $\boldsymbol{y}^{\gamma} \boldsymbol{P}^{*} \boldsymbol{y}$ constant), the mating preference phenotype obtaining the largest selective advantage is one in which each male trait is weighted in proportion to its covariance with expected progeny viability (namely, when the vectors $y$ and cov $\left\{z, \bar{w}^{\prime}(z)\right\}$ are parallel). Note that the individual covariances, $\operatorname{cov}_{i}\left\{z_{i}, \bar{w}^{\prime}(z)\right\}$, may be negative and yet lead to an advantage to mate choice, so long as the sign of the corresponding weight, $y_{i}$, is also negative. The elements of the covariance vector thus provide a measure of the relative strength of selection promoting the evolution of a mating preference for the separate male characters. Below I show that this can be quite different from the relative strength of direct selection on the male characters themselves.

In order to evaluate the effect of genetic covariation on the magnitude and direction of the force promoting the origin of a novel mating preference, an explicit formulation of $\bar{w}^{\prime}(z)$ is needed. This can be obtained by assuming that phenotypic values for the characters, $z_{1}, \ldots, z_{n}$, have a multivariate normal distribution and are subject to Gaussian optimising selection (as previously defined). If $p^{\prime}\left(z^{\prime} \mid z\right)$ is the probability density of progeny phenotypes, $z^{\prime}$, within families sired by a male of phenotype $z$, then the expected viability 
of these progeny is, by definition,

$$
\bar{w}^{\prime}(z)=\int p^{\prime}\left(z^{\prime} \mid z\right) w\left(z^{\prime}\right) d z^{\prime} .
$$

(Naturally, if selection is sex-limited, equation (11) applies only to male offspring.) Let $\overline{\boldsymbol{z}}^{\prime}(\boldsymbol{z})$ denote the vector of mean phenotypes within families sired by a male of phenotype $z$. Under the assumption of additive allelic effects, $\bar{z}^{\prime}(\boldsymbol{z})$ is obtained from the multivariate generalisation of the traditional equation of quantitative genetics:

$$
\bar{z}^{\prime}(\boldsymbol{z})-\overline{\boldsymbol{z}}=\boldsymbol{G} \boldsymbol{P}^{-1}\left(\frac{\boldsymbol{z}+\bar{z}_{f}}{2}-\bar{z}\right) .
$$

The term on the left of equation (12) expresses the vector of progeny means as a deviation from the vector of mean phenotypes in the parental generation. The term in brackets on the right is the expected midparent deviation of matings involving a male of phenotype $z_{\text {, where }} \bar{z}_{f}$ denotes the expected phenotype of a mating female. Under the assumption of random mating, $\bar{z}_{f}=\bar{z}^{*}$ if both males and females are subject to viability selection, whereas $\bar{z}_{f}=\bar{z}$ if selection is limited to males. The effect of mate choice on the expected phenotypic values of progeny is clarified by rearranging equation (12), using equation (4):

$$
\overline{\boldsymbol{z}}^{\prime}(\boldsymbol{z})-\overline{\boldsymbol{z}}=\Delta \overline{\boldsymbol{z}}+\frac{1}{2} \boldsymbol{G} \boldsymbol{P}^{-1}\left(\boldsymbol{z}-\overline{\boldsymbol{z}}^{*}\right) .
$$

From (13) it can be seen that the expected phenotype of a given male's progeny deviates from the mean of the parental generation by a sum of two terms. The first, $\Delta \bar{z}$, is the response of the overall population to viability selection on the parents. The second, $\frac{1}{2} \boldsymbol{G} \boldsymbol{P}^{-1}\left(\boldsymbol{z}-\bar{z}^{*}\right)$, represents the effect of mate selection owing to the deviation of a particular sire from the mean of mating males; it is also equal to the vector of deviations of the progeny mean phenotypes from the mean of their own generation, since the latter is given by the sum: $\bar{z}+\Delta \bar{z}$. Note that the effect of sex-uniform versus sex-limited selection on the progeny means is confined to the term $\Delta \bar{z}$, as defined in (4).

If one assumes that the distribution of phenotypic values within families sired by a given male is approximately multivariate normal, with covariance matrix denoted $\boldsymbol{A},(13)$ can be used to compute expected progeny viability. Using (1) and (13), the integral in (11) is found to be

$$
\overline{\boldsymbol{w}}^{\prime}(\boldsymbol{z}) \propto \exp \left\{-\frac{1}{2}(\boldsymbol{z}-\boldsymbol{\theta})^{T} \boldsymbol{B}(\boldsymbol{z}-\boldsymbol{\theta})\right\}
$$

where,

$$
\begin{gathered}
\boldsymbol{\theta}=\overline{\boldsymbol{z}}^{*}-2 \boldsymbol{P} \boldsymbol{G}^{-1}(\overline{\boldsymbol{z}}+\Delta \overline{\boldsymbol{z}}) \\
\boldsymbol{B}=\frac{1}{4}\left(\boldsymbol{G} \boldsymbol{P}^{-1}\right)^{T} \boldsymbol{A}^{-1}\left(\boldsymbol{A}^{-1}+\boldsymbol{W}\right)^{-1} \boldsymbol{W}\left(\boldsymbol{G} \boldsymbol{P}^{-1}\right) .
\end{gathered}
$$

If selection acts on all the traits, the symmetric matrix, $\boldsymbol{B}$, is positive definite, and describes a multivariate Gaussian fitness surface for the function $\bar{w}^{\prime}(z)$. This means that maximum offspring viability is found only among progeny of males whose vector of phenotypic values equals $\boldsymbol{\theta}$. In a population at its evolutionary optimum for all characters, where $\overline{\boldsymbol{z}}=\overline{\boldsymbol{z}}^{*}=\Delta \overline{\boldsymbol{z}}=\mathbf{0},(15 \mathrm{a})$ reveals that the phenotype of the optimum mate is the zero vector, that is, $\boldsymbol{\theta}$ corresponds both to the optimum under viability selection and to the mean of the population. However, in a population that deviates from the selective optimum for one or more characters, the most fit males no longer sire the most fit offspring, since under these conditions all the elements of $\boldsymbol{\theta}$ are typically non-zero. Rather, the optimum mate deviates from the viability optimum not only for those characters that themselves deviate from their optima, but also for any other genetically correlated characters (fig. 1). Hence, it may frequently be wrong to assume that adaptive mate choice corresponds to mating with the most viable males in a population.

This explicit model for the relationship between paternal phenotypes and progeny fitness

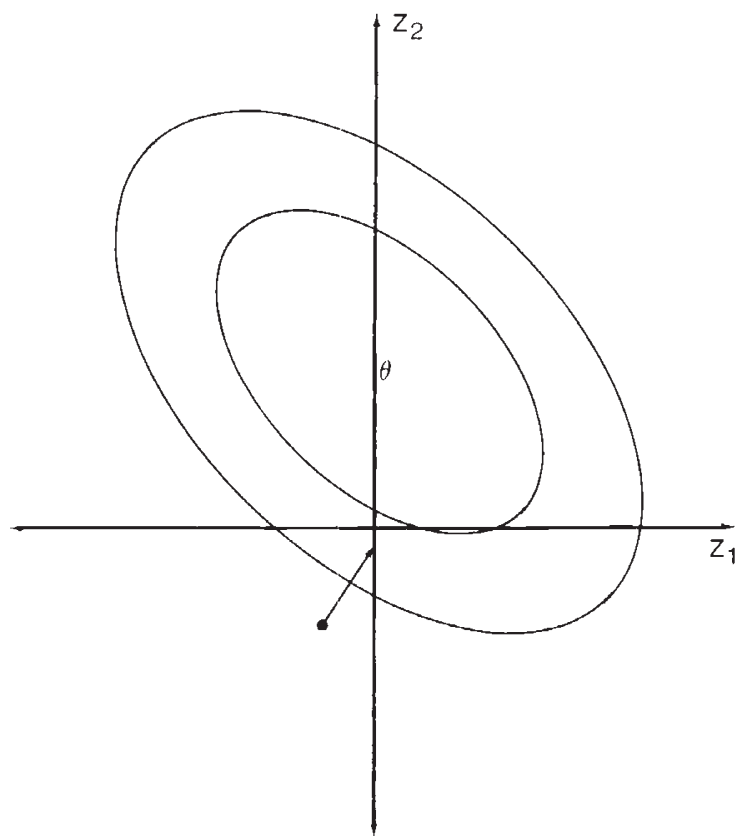

Figure 1 Offspring viability mapped over phenotypic space for two correlated traits, $z_{1}$ and $z_{2}$, in sires. Lines plot isopleths of the surface of expected progeny viability, $\bar{w}^{\prime}\left(z_{1}, z_{2}\right)$. The pair of phenotypic values in a sire that maximises progeny viability is indicated by $\boldsymbol{\theta}$. The phenotypic mean of the population is indicated by a solid circle, and the arrow gives the direction of selection for female mating preferences. See text for the derivations. 
can be used to investigate the effect of ongoing viability selection on a force for the origin of a directional mating preference. Using (14) and (15), the covariance between sire phenotypes and progeny fitness can be evaluated explicitly:

$$
\begin{aligned}
\operatorname{cov}\left\{z, \bar{w}^{\prime}(z)\right\} & =\int p^{*}(z)\left(z-\bar{z}^{*}\right) \bar{w}^{\prime}(z) d z \\
& \propto\left(\boldsymbol{P}^{*}{ }^{1}+\boldsymbol{B}\right){ }^{1} \boldsymbol{B}\left(\boldsymbol{\theta}-\bar{z}^{*}\right) .
\end{aligned}
$$

Under weak selection this simplifies to the approximation,

$$
\operatorname{cov}\left\{z, \bar{w}^{\prime}(z)\right\} \propto \boldsymbol{P}^{*} \boldsymbol{B}\left(\boldsymbol{\theta}-\bar{z}^{*}\right) \cong-\frac{1}{2} \boldsymbol{G} \boldsymbol{W}(\bar{z}+\Delta \bar{z}) .
$$

Several features of (17) are notable. First, both the direction and magnitude of the vector, $\operatorname{cov}\left\{z, \bar{w}^{\prime}(z)\right\}$ changes over time, owing to evolution of the mean phenotypes in the population. As the phenotypic means approach equilibrium under viability selection, the force promoting evolution of a directional mating preference decreases, vanishing when $\bar{z}_{i}=0$ for all characters. However, so long as any single character deviates from its phenotypic optimum, genetic correlations lead to forces promoting mating preferences directed at a number of different characters. (The disappearance of the force when all characters are at equilibrium applies only to directional preferences such as the linear system assumed in deriving (17). Nonlinear, optimising mating preferences may be promoted even at equilibrium for all characters.) Second, if the individual covariances in (17) were ranked according to their absolute magnitudes, these ranks would represent the relative degree to which deterministic forces, acting in a particular population at a specific point in its evolutionary history, favour the origin of a mating preference directed at any particular trait. Because of the role of the genetic covariance in determining these ranks, the ranking of a set of characters according to their approriateness as objects of sexual selection will typically not correspond to their ranking according to the intensity of natural selection on them, since the latter is purely phenotypic in determination (Lande, 1979; Lande and Arnold, 1983). Thus, traits that are subject to intersexual selection cannot, a priori, be assumed ever to have been subject to any other form of natural selection. This is the subject of the following section, which deals with neutral characters as potential mate choice criteria.

\section{(ii) Origin of an "arbitrary" mating preference}

There are two general situations in which a mating preference directed at an otherwise selectively neutral character might originate in a population. The first is when heritable characters that are important to progeny fitness cannot be observed at the time that females choose mates. Examples of this include larval characters in holometabolous insects and characters that are expressed only in females. In such cases, an otherwise selectively neutral trait that happens to be assessable by females during courtship may be useful in mate choice, so long as it is genetically correlated with other unobservable but selectively important characters. The second situation is that in which a neutral character may in fact be a better predictor of progeny fitness than are the directly selected characters themselves. This can occur if the neutral trait is genetically correlated with one or more selectively important characters, but is itself less subject to non-genetic causes of variation.

I shall analyse the conditions for the origin of a mating preference directed at an "arbitrary" neutral character by considering a simplified twocharacter case of the previous model. One of the characters, denoted $x$, is neutral with respect to viability selection but is genetically correlated with a second character, $z$, that is subject to Gaussian viability selection (i.e., $\boldsymbol{W}$ in equation (1) is a $2 \times 2$ matrix having only one non-zero entry). Under these assumptions, equation (16) reduces to,

$$
\left[\begin{array}{l}
\operatorname{cov}\left\{z, \bar{w}^{\prime}(z, x)\right\} \\
\operatorname{cov}\left\{x, \bar{w}^{\prime}(z, x)\right\}
\end{array}\right] \propto \boldsymbol{P}^{*} \boldsymbol{P}^{-1}\left[\begin{array}{l}
g_{z z} \\
g_{z, x}
\end{array}\right](\bar{z}+\Delta \bar{z}),
$$

where $g_{z z}$ and $g_{z x}$ are elements of the genetic covariance matrix, $\boldsymbol{G}$. So long as the selected character is undergoing directional selection and the two traits are genetically correlated $(\bar{z} \neq$ $0, g_{z x} \neq 0$ ), there is a force of selection for mating preference directed at the otherwise selectively neutral character. Furthermore, the direction of the vector of covariances between the two characters and progeny fitness remains constant over time (which would not be true were both characters subject to direct selection). This means that the relative value of the two characters as mate choice criteria remains constant, and that throughout the period of evolution of the selected character, there is a force for the origin of a mating preference directed at any detectable, genetically correlated, but otherwise neutral trait. Whether the mating preference will favour the elaboration of the neutral character or its diminution will depend 
both on the sign of the genetic covariance, $g_{z x}$, and the direction in which the selected character itself is evolving.

It is possible for a selectively neutral character actually to be a better mate choice criterion than is a correlated non-neutral character, even if the latter can be assessed by females. Consider two alternative linear mating preferences, one in which females choose mates solely on the basis of the selected trait $z$, the other in which females discriminate among potential mates to the same degree, but solely on the basis of the neutral character $x$. Thus, the alternative female preference phenotypes could be written: $y_{z}=\left(a / \sigma_{z}^{*}, 0\right)^{T}, y_{x}=\left(0, a / \sigma_{x}^{*}\right)^{T}$. The constant, $a$, is the weight both types of females apply to the deviation of a potential mate from the mean of available males, while the phenotypic standard deviations, $\sigma_{z}^{*}$ and $\sigma_{x}^{*}$, standardise these deviations and insure that both types of females discriminate equally among potential mates. Substituting the two alternative preferences into (10) reveals that mate choice based on the neutral trait yields a larger increase in expected progeny fitness if the following inequality holds:

$$
\operatorname{cov}\left\{z, \bar{w}^{\prime}(z, x)\right\} / \sigma_{z}^{*}<\operatorname{cov}\left\{x, \bar{w}^{\prime}(z, x)\right\} / \sigma_{x}^{*} .
$$

Under weak natural selection $\left(\boldsymbol{P}^{*} \cong \boldsymbol{P}\right),(18)$ indicates that the ratio of the two covariances is approximately $g_{z z} / g_{z x}$, and using this, the inequality in (19) approximates to

$$
h_{z}<\rho_{z x} h_{x}
$$

where $h_{z}$ and $h_{x}$ are the square roots of the respective heritabilities of the two traits (defined here as the coefficients of regression of progeny mean phenotypes on the average phenotype of their parents), and $\rho_{z x}$ is the correlation of additive genotypic values between them. Equation (20) is the traditional equation for "indirect selection", which gives the conditions under which artificial selection on a correlated character provides a better response than does selection directly on the character whose improvement is desired (Falconer, 1981, p. 320). Again, the similarity between sexual and artificial selection is evident.

\section{DYNAMIC MODELS}

In the previous section, I described the conditions under which a mating preference directed at an otherwise selectively neutral character might enter a population that previously lacked it. Here, I develop a dynamic model to investigate the outcome of the evolution of such an "indirect" preference, given that it has successfully entered a population. The previous models suggest that this is most likely to occur if the neutral "criterion" character is genetically correlated with other traits that are subject to direct, directional viability selection. I therefore shall consider the evolution of a set of three traits: first, a "selected" character, denoted $z$, that is subject solely to viability selection; second, a "criterion" character, denoted $x$, that is subject solely to sexual selection via female choice, but that is genetically correlated with the "selected" character $z$; and third, the female mating preference, denoted $y$, that is not subject to direct selection but evolves via its genetic correlations with the two other traits. I shall consider two patterns of viability selection, one in which the selected character $z$ is expressed only in males, the other in which viability selection affects both sexes.

\section{(i) Equilibrium under sex-limited viability selection}

The two selected characters, $z$ and $x$, are assumed to be expressed only in males, having phenotypic variances $\sigma_{z}^{2}$ and $\sigma_{x}^{2}$, respectively, and phenotypic covariance $C_{z x}$. The phenotypic variance of the female mating preference is denoted $\sigma_{y}^{2}$. The pattern of genetic covariation is described by the $3 \times 3$ matrix $\boldsymbol{G}$, whose elements are denoted $g_{i j}, i, j=$ $z, x, y$. Multivariate normality is assumed for the distributions of genotypic values and environmental deviations. The marginal distributions of the phenotypic values for the three separate characters are denoted $p_{z}(z), p_{x}(x)$ and $q(y)$, respectively. Selection is assumed to be sufficiently weak that the pattern of genetic variation remains approximately constant over the evolutionary timespan considered.

Viability and sexual selection are assumed to operate sequentially. If the two male characters are phenotypically correlated, viability selection on character $z$ produces an observable change in the mean of character $x$; likewise, subsequent sexual selection on character $x$ further changes the mean of character $z$. In both episodes of selection, the observable selection differential on the unselected character is equal to the selection differential on the directly selected character multiplied by the coefficient of regression of the unselected on the selected trait. Using this information, the total selection differentials on the two male characters, denoted $S_{z}^{(t)}$ and $S_{x}^{(t)}$, can be written as a sum of 
separate components from the two sequential episodes:

$$
\begin{aligned}
& S_{z}^{(1)}=S_{z}^{(v)}+\frac{C_{z x}^{*}}{\sigma_{x}^{* 2}} S_{x}^{(s)}, \\
& S_{x}^{(1)}=\frac{C_{z x}}{\sigma_{z}^{2}} S_{z}^{(v)}+S_{x}^{(s)},
\end{aligned}
$$

where the superscripts $(v)$ and $(s)$ denote viability and sexual selection, respectively. The asterisks in (21a) indicate phenotypic covariance and variance following viability selection, since selection itself alters the pattern of phenotypic covariation (see below).

Evolutionary change in the mean phenotypes is given by the equation:

$$
\left[\begin{array}{c}
\Delta \bar{z} \\
\Delta \bar{x} \\
\Delta \bar{y}
\end{array}\right]=\frac{1}{2} G\left[\begin{array}{c}
I_{z} \\
I_{x} \\
0
\end{array}\right]
$$

where $I_{z}$ and $I_{x}$ are the selection gradients on the two male characters, calculated as,

$$
\left[\begin{array}{l}
I_{z} \\
I_{x}
\end{array}\right]=\left[\begin{array}{cc}
\sigma_{z}^{2} & C_{z x} \\
C_{z x} & \sigma_{x}^{2}
\end{array}\right]^{-1}\left[\begin{array}{c}
S_{z}^{(t)} \\
S_{x}^{(t)}
\end{array}\right] .
$$

The factor of one-half accounts for sex-limitation of viability and sexual selection.

Gaussian viability selection is assumed, with $w(z)$, the viability of a male having phenotypic value $z$, given by,

$$
w(z)=\exp \left(-z^{2} / 2 \omega^{2}\right) .
$$

The effect of this type of selection on the joint distribution of the two male characters can be obtained from equation ( $3 a$ ), where the matrix $\boldsymbol{W}$ has the single non-zero entry, $W_{z z}-1 / \omega^{2}$. Solving for $\boldsymbol{P}^{*}$ yields,

$$
\begin{aligned}
& C_{z x}^{*}=\omega^{2} C_{z x} /\left(\omega^{2}+\sigma_{z}^{2}\right) ; \\
& \sigma_{x}^{*^{2}}=\sigma_{x}^{2}-C_{z x}^{2} /\left(\omega^{2}+\sigma_{z}^{2}\right) .
\end{aligned}
$$

The selection differential on character $z$ owing to viability selection alone is found to be

$$
S_{z}^{(v)}=\frac{\int z p_{z}(z) w(z) d z}{\int p_{z}(z) w(z) d z}-\bar{z}=\frac{-\sigma_{z}^{2}}{\omega^{2}+\sigma_{z}^{2}} \bar{z} .
$$

Mating preferences are modelled using the three preference functions originally introduced by Lande (1981). These are (1) "psychophysical" preferences, in which individual females have motononically increasing (or decreasing) preference for males having increasing values of $x$ :

$$
\Psi(x \mid y) \propto \exp (x y),
$$

(2) relative unimodal preferences, in which individual females most prefer males whose phenotype deviates from the mean of available males by a quantity $y$ :

$$
\Psi(x \mid y) \propto \exp \frac{-\left\{x-\left(\bar{x}^{*}+y\right)\right\}^{2}}{2 \nu^{2}}
$$

and (3) absolute unimodal preferences, in which individual females most prefer males whose phenotype equals some value $y$, independent of the distribution of available males:

$$
\Psi(x \mid y) \propto \exp \frac{-(x-y)^{2}}{2 \nu^{2}} .
$$

Psychophysical preferences are similar to the linear preferences discussed in the previous section, producing the same sexual selection differential on males while preserving normality of the phenotypic distribution. In both the relative and absolute unimodal preferences, the parameter $\nu$ measures the rate at which preference decreases as males increasingly deviate from the most preferred type. It can be regarded as a measure of female "choosiness".

Females choose mates from the distribution of males following viability selection. Denoting this distribution $p_{x}^{*}(x)$, it has mean $\bar{x}^{*}=\bar{x}+S_{x}^{(v)}$, and variance $\sigma_{x}^{* 2}$, defined above. The relative preference of a female of phenotype $y$ for a male of phenotype $x$ is defined as,

$$
\Psi^{*}(x \mid y)=\frac{\Psi(x \mid y)}{\int p_{x}^{*}(x) \Psi(x \mid y) d x}
$$

The relative fitness of a male under sexual selection, denoted $w^{*}(x)$, is obtained by averaging relative preferences over the distribution of female phenotypes:

$$
w^{*}(z)=\int q(y) \Psi^{*}(x \mid y) d y .
$$

If the three specified preference functions are substituted into (28), the sexual selection differential on the criterion trait is found to be,

$$
\begin{aligned}
S_{x}^{(s)} & =\int x p_{x}^{*}(x) w^{*}(x) d x-\bar{x}^{*} \\
& =\alpha \sigma_{x}^{* 2}\left(\bar{y}-\varepsilon \bar{x}^{*}\right) .
\end{aligned}
$$


The parameters $\alpha$ and $\varepsilon$ distinguish the three types of preference function, with $\alpha=1$ with psychophysical preferences and $1 /\left(\nu^{2}+\sigma_{x}^{* 2}\right)$ with unimodal preferences; while $\varepsilon=1$ with absolute unimodal preferences and zero otherwise.

The viability and sexual selection differentials obtained in (26) and (30) can now be substituted into (21). Using (25) the total selection differentials on the two male characters are found to be

$S_{z}^{(t)}=\left(\frac{-\sigma_{z}^{2}+\alpha \varepsilon C_{z x} C_{z x}^{*}}{\omega^{2}+\sigma_{z}^{2}}\right) \bar{z}+\alpha C_{z x}^{*}(\bar{y}-\varepsilon \bar{x}) ;$

$S_{x}^{(t)}=\left(\frac{-C_{z x}+\alpha \varepsilon C_{z x} \sigma_{x}^{* 2}}{\omega^{2}+\sigma_{z}^{2}}\right) \bar{z}+\alpha \sigma_{x}^{* 2}(\bar{y}-\varepsilon \bar{x})$.

Equilibrium under this model occurs only when the total selection differentials on both characters are simultaneously zero, provided that none of the characters are completely correlated genetically ( $\boldsymbol{G}$ nonsingular). This occurs only when $\bar{z}=0$ and $\bar{y}=$ $\varepsilon \bar{x}$. There thus exists a line of equilibria in which the mean of the viability character lies at the viability optimum, while the criterion character may take on any value so long as it matches the overall female preference in the population (i.e., is not subject to any directional force from female mating preferences). This equilibrium state differs from that of Lande (1981) in that it involves no "tradeoff" between competing forces of viability and sexual selection on males. This is because, under the assumption that the two male characters are not perfectly correlated genetically, there is always some genetic variance available for them to evolve independently. The criteria for stability of this line of equilibria are quite strict, however; they will be discussed in a later section.

\section{(ii) Equilibrium under sex-uniform viability selection}

In this model I assume that the character $z$ is identically distributed in both males and females, and likewise is subject to the same force of viability selection. The model is identical to that for sexlimited selection except that viability selection on females now alters the distributions both of the viability character and the mating preference in females. If the selection differentials for the two traits in females are denoted $S_{z}^{(f)}$ and $S_{v}^{(f)}$, they can be computed in the same way as were the selection differentials on males:

$$
\begin{aligned}
& S_{z}^{(f)}=S_{z}^{(v)} ; \\
& S_{y}^{(f)}=\frac{C_{z y}}{\sigma_{z}^{2}} S_{z}^{(f)} .
\end{aligned}
$$

Thus, the selection differentials owing to viability selection on character $z$ are identical in males and females, and an observed change in the mean of the female mating preference is created, which is proportional to the phenotypic regression of $y$ on $z$ in females.

The selection differentials in males are unchanged, except that the mean female mating preference, as it appears in equation (31), is now equal to the mean following viability selection: $\bar{y}^{*}=$ $\bar{y}+S_{y}^{(f)}$.

Evolutionary change in the mean phenotypes for the three characters is given by

$$
\left[\begin{array}{c}
\Delta \bar{z} \\
\Delta \bar{x} \\
\Delta \bar{y}
\end{array}\right]=\frac{1}{2} G\left[\begin{array}{c}
I_{z}^{(m)}+I_{z}^{(f)} \\
I_{x}^{(m)} \\
I_{y}^{(f)}
\end{array}\right]
$$

where $I_{z}^{(m)}, I_{x}^{(m)}$ again are the selection intensities on the two male characters, defined in (23), and $I_{z}^{(f)}, I_{y}^{(f)}$ are the intensities in females, computed as

$$
\left[\begin{array}{c}
I_{z}^{(f)} \\
I_{y}^{(f)}
\end{array}\right]=\left[\begin{array}{cc}
\sigma_{z}^{2} & C_{z y} \\
C_{z y} & \sigma_{y}^{2}
\end{array}\right]^{-1}\left[\begin{array}{c}
S_{z}^{(f)} \\
S_{y}^{(f)}
\end{array}\right] .
$$

As in the sex-limited model, equilibrium occurs only when the selection differentials are all zero, which again obtains only when $\bar{z}=0$, and $\bar{y}=\varepsilon \bar{x}$. Thus, the sex-limited and sex-uniform selection models are identical as far as their equilibria are concerned.

\section{(iii) Stability of the equilibria}

Stability analysis proceeds using the transformation matrix, $\boldsymbol{M}$, defined by,

$$
\left[\begin{array}{c}
\Delta \bar{z} \\
\Delta \bar{x} \\
\Delta \bar{y}
\end{array}\right]=\boldsymbol{M}\left[\begin{array}{c}
\bar{z} \\
\bar{x} \\
\bar{y}
\end{array}\right] .
$$

Assuming weak selection $\left(\omega^{2}, \nu^{2} \gg \sigma_{z}^{2}, \sigma_{x}^{2}, \sigma_{y}^{2}\right)$, and using (22) and (23), $\boldsymbol{M}$ is approximately given by,

$$
\boldsymbol{M}=\frac{1}{2} \boldsymbol{G}\left[\begin{array}{ccc}
-2 k / \omega^{2} & \alpha \varepsilon C_{z x} / \omega^{2} & -\alpha C_{z x} / \omega^{2} \\
\alpha \gamma / \omega^{2} & -\alpha \varepsilon & \alpha \\
0 & 0 & 0
\end{array}\right],
$$

where, $\gamma=\varepsilon C_{z x}, k=\frac{1}{2}$, if viability selection is sexlimited, and $\gamma=\varepsilon C_{z x}-C_{z y}, k=1$, if selection acts on both males and females. The matrix $\boldsymbol{M}$ has three eigenvalues, one of which is zero and indicates neutrality of the line of equilibria. The others, denoted $\lambda_{+}$and $\lambda_{-}$, are roots of the quadratic equation:

$$
0=-\lambda^{2}+A \lambda+D
$$


where,

$$
\begin{gathered}
A=\frac{1}{2 \omega^{2}}\left\{-2 k g_{z z}+\alpha g_{z x}\left(\gamma+\varepsilon C_{z x}\right)-\alpha C_{z x} g_{z y}+\right. \\
\left.\alpha \omega^{2}\left(g_{x y}-\varepsilon g_{x x}\right)\right\} \\
D=\frac{\alpha k}{2 \omega^{2}}\left[\left(g_{z z} g_{x y}-g_{z x} g_{z y}\right)-\varepsilon\left(g_{x x} g_{z z}-g_{z x}^{2}\right)\right]
\end{gathered}
$$

The mean phenotypes evolve in the plane determined by the two eigenvectors corresponding to the roots, $\lambda_{+}$and $\lambda$. Since change over time is determined by a linear combination of $\left(1+\lambda_{+}\right)^{t}$, convergence to a point on the line of equilibria (i.e., stability) requires that $\left|1+\lambda_{t}\right|<1$, where $|\cdot|$ denotes the norm of a number that may be real or complex. Under weak selection, the psychophysical model always yield real eigenvalues, but the unimodal preference models may not.

Stability of the line of equilibria depends on the parameters in equation (37) in the following way. If $D>0$, the eigenvalues are always real and the line of equilibria always unstable. For relative preferences $(\varepsilon=0)$, the criterion $D<0$ reduces to

$$
\rho_{x y}<\rho_{z x} \rho_{z y},
$$

where the $\rho_{i j}$ are the genetic correlation coefficients: $g_{i j} / \sqrt{g_{i i} g_{j j}}$. In the absence of pleiotropic effects from character $z$ on the mating preference, $\rho_{z y}$, will be less than $\rho_{x y}$, since it is through the latter, generated by nonrandom mating, that the former arises. In this case, the condition in (38) cannot be met, and the population will evolve away from the line of equilibria. Thus, relative mating preferences, whether directional or optimising, are unlikely to produce a stable evolutionary outcome. Note that this condition is independent of whether or not viability selection is limited to males.

If $D<0$, the eigenvalues may be real or complex. Under the assumption of weak selection, $|\lambda| \ll 1$, and the evolutionary rates are approximated by $(1+\lambda)^{t} \cong \exp (t \lambda)$. Given this approximation, the line of equilibria will be stable if $A<0$. Only absolute preferences are likely to meet the condition $D<0$, and for them $A$ will be negative if, approximately,

$$
\frac{\nu^{2}}{\omega^{2}}>\frac{g_{x y} \cdots g_{x x}}{2 k g_{z z}} \text {. }
$$

This criterion is similar to that of Lande (1981) for absolute mating preferences, and reduces to his in the limit where $z$ and $x$ are identical. The factor of $2 k$ in (39) indicates that with absolute mating preferences, populations are more likely to evolve to and remain on their line of equilibria if both males and females are subject to viability selection than if selection is limited to males.

\section{DISCUSSION}

Fisher (1915) suggested that mating preferences for many characters initially arise owing to natural selection on offspring, mediated by genetic correlations between selectively neutral criterion chracters and other characters that affect fitness directly. I have presented here a set of mathematical models that elucidate Fisher's theory as it applies to quantitative characters that are subject to optimising viability selection.

In certain situations selectively unimportant characters may become the criteria used in mate choice. Most obvious among these are cases in which characters that are subject to direct selection cannot be evaluated by females, either because they are not observable at the time that females choose mates, or because females lack the sensory discrimination needed to evaluate them. For example, selective forces on larval characters in holometabolous insects may be very strong, yet virtually none of these traits occur in reproductive adults. Likewise, characters expressed only in females may significantly contribute to overall progeny fitness. Genetic correlations between characters subject to selection in juveniles or females and traits expressed in reproductive males provide a basis for the evolution of "indirect" mating preferences. Indirect preferences may also be involved when females discriminate on the basis of male vocalisations prior to physically encountering potential mates. Several authors have suggested that body size is important to fitness in anuran amphibians and postulate that female anurans use male call characteristics as an indirect estimator of body size when approaching breeding aggregations (Licht, 1976; Wilbur, et al., 1978; Ryan, 1980; Fairchild, 1981).

The likelihood that a character will become the object of a mating preference is less related to its direct effects on fitness than to its value as a predictor of breeding values for fitness. In the extreme, selectively neutral characters may be more likely to become the objects of sexual selection than are selectively important traits, even though the latter can also be assessed by females. I see two ways in which this might occur. First, a neutral character may be less subject to environmental variation during development than are selectively important characters with which it is correlated. Second, a 
neutral character may be less subject to error in its assessment by females. For example, two traits may possess equal genetic variance but differ in the sensory modality by which they are ascertained. Mating preferences directed at the character perceived by the more acute of these two senses will correlate more highly with progeny fitness. This is analogous to the effect of measurement error on heritability estimates made by quantitative geneticists. In both cases error in measurement combines with environmental variance to reduce the regression of offspring on parental phenotypes (Falconer, 1981). Geneticists use multiple measures on the same individual to reduce the effect of measurement error on heritability. Perhaps the repetition of single elements during prolonged bouts of courtship, a common phenomenon in animals, fulfills a similar role in mate choice.

If an otherwise selectively neutral character does come to be the object of a mating preference, the dynamic models indicate that its evolutionary path may be quite complex. A similarity between the present model and other sexual selection models is the existence of a line of neutral equilibria wherein female mating preferences and the male characters at which they are directed are maintained in a state of mutual balance, with an infinity of possible degrees of elaboration for the male character (Lande, 1981; Kirkpatrick, 1982). Random evolution of both the male and female characters could occur along this line, while perturbations away from the line could lead either to a return to equilibrium if the line is stable or to runaway evolution if it is not. In previous models, this line of neutral equilibria entailed trade-offs between conflicting forces of viability and sexual selection on males. In the present models, however, the characters subject to these separate forces are not perfectly coupled, and it is possible for the sexually selected trait to evolve a high degree of elaboration without preventing the viability character from evolving to its optimum value. This is only likely to happen, however, if females choose mates according to absolute standards, since this tends to exert a stabilising force on evolution of the secondary sexual character. Otherwise, the "runaway process" is likely, and both the viability and sexual characters will evolve away from their respective equilibria.

A weakness of the present model is its inability to evaluate the degree to which the combined forces of sexual and viability selection may alter the pattern of genetic covariation itself. If the genetic and ecological conditions for instability are met, it is unlikely that runaway evolution can be sustained. Rather, the limit to selection determined by the available genetic variance for the evolving characters will be critical in deciding the outcome of the evolutionary process. The analysis of the dynamic models cannot therefore be interpreted as a prediction of the outcome of evolution of male characters and female mating preferences. Rather, it reveals that the conditions for a simple, predictable outcome are extremely strict.

Two results suggest that it is vital in studies of sexual selection to distinguish between direct selection and its indirect effects. First of these is the observation that deterministic forces may cause traits to become the objects of mate choice even though they are not subject to direct viability selection. Second are the differences both in the types of equilibria that occur and in their stability when quantitative traits are simultaneously subject to both sexual and viability selection (as in Lande's model) versus when these forces are partially uncoupled, acting on separate but correlated characters. Although the concept of indirect mate choice criteria is widely used in discussions of sexual selection, its consequences are not widely appreciated.

This suggests that some approaches currently used in studies of female choice are unlikely to be fruitful in identifying the selective forces involved. Often, mate choice criteria are inferred from observed differences in the distribution of single characters in mated and unmated individuals. For instance, positive correlations between mating success and body size have been observed in a variety of species (LeBeouf, 1974; Downhower and Brown, 1980; Howard, 1980; Ryan, 1980; Schmale, 1981). It is common for an association between the identified character and male fitness to be suggested, or if such an inference is difficult to support, to invoke Fisher's process whereby a mating preference will be maintained solely through its association with high mating success in male progeny.

The hazard of this approach is the possibility of seriously misinterpreting phenotypic data. First, phenotypic correlations may lead to observed changes in the distribution of traits which are not themselves the objects of selection. If the objects of female choice are to be identified, a multivariate method such as that suggested by Lande and Arnold (1983) is needed in order to distinguish between direct and indirect effects of mate choice. Second, if a character is in fact identified as an object of mating preference, it does not necessarily follow that it will have any direct relation to fitness beyond its influence on mating success. When females use indirect mate choice criteria, an 
association between a preferred character and progeny viability can only be identified by observing the relationship between progeny survivorship and the phenotype of their sire. Finally, conflicts between the directions of sexual and viability selection may lead to negative correlations between preferred characters and viability even when no net force of directional selection on mating preferences is present. These complications do not depend on the existence of unusual modes of inheritance or complex patterns of selection, for they arise, as in the present models, merely from the existence of pleiotropy among quantitative traits, a common if not universal phenomenon (Wright, 1968).

The present models confirm that when males contribute nothing except their genes to their progeny, indirect forces of selection for female choice may indeed exist. Beyond this simple level of analysis, however, the arguments that typify the "good genes" view (e.g., Thornhill and Alcock, 1983) do not bear out. The characters we find to be the objects of female choice need not ever have been important to survival, nor does genetic reasoning provide a basis for expecting that females should generally be most attracted to the most vigorous males in a population. Furthermore, if the characters to which females respond in choosing mates do not directly affect survival, the outcome of evolution may be virtually unpredictable. The central flaw in the "good genes" approach is the assumption that indirectly-evolving mating preferences respond to ecological pressures in the same way as do directly-selected survival characters. The "environment" in which such mating preferences evolve, however, is the genetic rather than the ecological milieu. Only by direct study of this genetic "environment" can we expect ever to achieve a satisfactory understanding of patterns of mate choice.

Acknowledgements 1 thank S. Arnold, M. Kirkpatrick, R. Lande and $M$. Wade for comments and suggestions on the manuscript, and R. Lande for helpful advice on the dynamic analysis.

\section{REFERENCES}

BATESON, P., ed. 1983. Mate Choice. Cambridge University Press, Cambridge.

BOAKE, C. 1985. Genetic consequences of mate choice: a quantitative genetic method for testing sexual selection theory. Science, 227, 1061-1063.
BORGIA, G. 1979. Sexual selection and the evolution of mating systems. In Blum, M. F. and Blum, N. A. (eds.) Sexual Selection and Reproductive Competition in Insects, Academic Press, New York, pp. 19-80.

BUIMER, M. (i. 1980. The Malhematical Theory of Quantitative Genetics. Clarendon Press, Oxford.

DOWNHOWER, J. F. ANI BROWN, L. 1980. Mate preferences of female mottled sculpins, Cottus bairdi. Animal Behaviour, 28, 728-734.

FAIR(HILD, L. 1981. Mate selection and behavioral thermoregulation in Fowler's toads. Science, 212, 950-951.

FALCONER, D. S. 1981. Introduction to Quantitative Genetics, 2nd Edn. Longman Group Ltd., London.

FISHFR, R. A. 1915. The evolution of sexual preference. Eugenics Review, 7, 184-192.

FISHER, R. A. 1958. The Genetical Theory of Natural Selection, 2nd Edn. Dover, New York.

HFISL.ER, I. L. 1984. A quantitative genetic model for the origin of mating preferences. Evolution, 38, 1283-1295.

HOWARD, R. D. 1980. Mating behaviour and mating success in wood frogs, Rana sylvatica. Animal Behaviour, 28, 705-716.

KIRKPATRIC'K, M. 1982. Sexual selection and the evolution of female choice. Evolution, 36, 1-12.

LANDE, R. 1979. Quantitative genetic analysis of multivariate evolution, applied to brain: body size allometry. Evolution, $33,402-416$.

LANDE, R. 1980. Sexual dimorphism, sexual selection and adaptation in polygenic characters. Evolution, 34, 292-305.

I.ANDE, R. 1981. Models of speciation by sexual selection on polygenic traits. Proceedings, National Academy of Sciences, USA, 78, 3721-3725.

LANIDE, R. AND ARNOLD, S. J. 1983. The measurement of selection on correlated characters. Evolution, 37, 12101226.

LEBOEUF, B. J. 1974. Male-male competition and reproductive success in elephant seals. American Zoologist, 14, 163-176.

I.ICHT, I. \& : 1976. Sexual selection in toads (Bufo americanus). Canadian J. Zoology, 54, 1277-1284.

O'DONALD, P. 1967. A general model of sexual and natural selection. Heredity, 22, 499-518.

O'donai. D, P. 1980. Genetic Models of Sexual Selection. Cambridge University Press, Cambridge.

PARTRIDGE, L. 1980. Mate choice increases a component of offspring fitness in fruit flies. Nature, 283, 290-291.

RYAN. M. J. 1980. Female mate choice in a Neotropical frog. Science, 209, 523-525.

S(:HAEFFER, S. W., BROWN, C. J. ANI) ANI)IRSON, W. W. 1984. Does mate choice affect fitness? Genetics, 107, S94.

SC:HMALE, M. C. 1981. Sexual selection and reproductive success in males of the bicolor damselfish, Eupomacentrus partitus (Pisces: Pomacentridae). Animal Behaviour, 29, $1172-1184$.

THORNHIL, R. ANI) ALCOCK, J. 1983. The Evolution of Insect Mating Systems. Harvard University Press, Cambridge, Massachusetts.

TRIVIERS, R. L. 1976. Sexual selection and resource-accruing abilitics in Anolis garmani. Evolution, 30, 253-269.

WILBER, H. M., RUBENSTIIIN, D. I. ANI) FAIRCHILD, L. 1978. Sexual sclection in toads: the roles of female choice and male body size. Evolution, 32, 264-270.

WITTHENBERGi:R, J. F. 1983. Tactics of mate choicc. In, Bateson, P. (ed.) Mate Choice, Cambridge University Press, Cambridge.

WRICill, s. 1968. Evolution and the Genetics of Populations, Vol. 1, University of Chicago Press, Chicago. 